\title{
Publisher Correction to: Corrections to Hawking radiation and Bekenstein-Hawking entropy of novel four-dimensional black holes in GaussBonnet gravity
}

\author{
Gu-Qiang $\mathrm{Li}^{1,2}$ (1) Jie-Xiong $\mathrm{Mo}^{1,2} \cdot$ Yi-Wen Zhuang ${ }^{2}$
}

Published online: 23 November 2021

(c) Springer Science+Business Media, LLC, part of Springer Nature 2021

\section{Correction to: General Relativity and Gravitation https://doi.org/10.1007/s10714-021-02863-7}

The publication of this article unfortunately contained a mistake: The formatting of the reference list was not correct and included some broken URLs.

The original article has been corrected.

Publisher's Note Springer Nature remains neutral with regard to jurisdictional claims in published maps and institutional affiliations.

The original article can be found online at https://doi.org/10.1007/s10714-021-02863-7.

$凶$ Gu-Qiang Li

ligq@lingnan.edu.cn

1 Institute of Theoretical Physics, Lingnan Normal University, Zhanjiang 524048, Guangdong, China

2 Department of Physics, Lingnan Normal University, Zhanjiang 524048, Guangdong, China 